\title{
Besprechung von Seidlitz' Fauna baltica ed. II.
}

Fauna baltica. Die Käfer der Ostseeprovinzen Rufslands von Dr. G. Seidlitz. Zweite neu bearbeitete Auflage. 1. Lieferung, Einleitung p. I-XL u. 1 lith. Taf., Gatt. p. 1-16, Arten p. 1-96.

Die 2te Auflage der Fauna baltica ist nach ähnlichen Principien bearbeitet wie die erste, $d . h$. sie ist nicht nur eine Compilation aus bekannten Werken, sondern sie beruht zum grofsen Theil auf eigenen Untersuchungen des Verfassers unter sorgfältiger Berücksichtigung der betreffenden Literatur bis auf die neueste Zeit; im Vergleich zur ersten Auflage ist sie in sofern bedeutend vermehrt, als sämmtliche im nördlichen Deutschland bis Berlin vorkommenden Arten und sämmtliche in Europa vorkommenden Gattungen und Untergattungen beschrieben und aufserdem die meisten europäischen Arten erwähnt und gruppirt sind.

Die Einleitung behandelt auf drei Bogen in besonderen Abtheilungen mehrere Capitel von allgemeinem Interesse, von denen mir namentlich das fünfte "die Systematik und ibre Bedeutung in der wissenschaftlichen Zoologie" und das sechste "die pädagogische und ethische Bedeutung der Entomologie" so beachtenswerth erscheinen, dafs dieselben im nächsten Hefte unserer Zeitschrift ganz zum Abdruck gebracht werden sollen. In No. 5 wird die Aufgabe des Systematikers als des Erforschers des natürlichen Systems behandelt, welches allmählig an Stelle des künstlichen Systems treten soll. Dr. Seidlitz spricht sich mit Entschiedenheit für die Aufrechterhaltung des Unterrichts in den alten Sprachen auf den höheren Schulen aus; sollten dieselben aber doch einmal verdrängt werden, so dürfte neben der Physik nur die Botanik, namentlich aber die Entomologie mit ihrem immensen Formenreichthum ${ }^{1}$ ) Ersatz liefern und zwar vorzüglich durch praktische Uebung der reinen Systematik nach synthetischer Methode; diese allein gewährt vor allen naturhistorischen Disciplinen die drei Erfordernisse des wahren Schulzweckes, nämlich: den Geist ohne jede Belastung des Gedächtnisses zu üben, ideale Zwecke zu

1) Derselbe ist selbst den Museumsvorständen, welche nicht Entomologen von Fach sind, noch immer viel zu wenig bekannt, wie die Ausführungen des Professor Landois auf der Wiesbadener Naturforscher-Versammlung zum Theil sehr deutlich bewiesen. 
verfolgen lehren und den Einblick in die Wissenschaft zu eröffnen, eines der idealen Güter der Menschheit. -

Die erste Lieferung bringt aufser der Einleitung (40 S.) eine Uebersicht der Gattungen und Arten der Laufkäfer und eines Theiles der Wasserkäfer, welche der Verfasser bereits vorher für Reitter's Bestimmungstabellen bearbeitet hatte.

Dals Procerus, Procrustes und die Schaar der übrigen CarabenGruppen als eine einzige natürliche Gattung Carabus betrachtet werden, ist sicherlich kein Fortschritt in der systematischen Entomologie; allerdings kommen wir nur langsam in der Erkenntnifs der natürlichen Gattungen vorwärts, aber gewifs nicht durch $\mathrm{Zu}$ sammenziehen aller bekannten Caraben in eine Gattung, sondern durch Vereinigung des natürlich zusammengehörenden in viele kleine Gruppen; dazu bildet das genaue Studium der Sculptur den Ausgangspunkt.

Wenn ich nun einerseits eine Gattung Orinocarabus aufgestellt habe, welche ein anderer sorgfältiger Forscher, wie Ganglbauer, in ihrem ganzen Umfange annimmt, so sehen wir den Verfasser Orinocarabus für eine natürliche Untergattung erklären, an deren Spitze Carabus convexus steht (welchen auch Géhin zu seiner Gattung Orcocarabus zieht), ebenso wie den sehr abweichenden Chamissonis, Weisei, die Verwandten des Ghilianii und die des Staehlini (nicht Staehlinii) Ad.; diese zieht nun Seidlitz ebenfalls zu Orinocarabus. $\mathrm{Ob}$ er weiter nach berühmten Mustern arbeitet, habe ich hier keine Veranlassung zu verfolgen; es genügt mir, auszusprechen, dafs ich Orinocarabus für eine natürliche Gruppe halte, ebenso natürlich wie die Staehlini-Gruppe des Caucasus-Gebietes; beide zu vereinigen scheint mir unthunlich. Wenn Seidlitz auf Carabus nemoralis eine eigene Untergattung mit einem neuen Namen (Archicarabus) begründet, so scheint mir dieselbe auf sehr schwachen Füfsen zu steben; denn in den zahlreichen Borstenpunkten an den Seiten des Halsschildes kann ich kaum mehr als ein specifisches Merkmal erblicken, weil monticola auch bisweilen einen Borstenpunkt jederseits mehr hat und die Mandibeln je nach der Haltung des Kopfes einen sehr verschiedenen Eindruck hervorbringen; aufserdem macht die Stellung dieser französisch-italienischen Art zwischen sibirischen und osteuropäischen Arten durchaus keinen natürlichen Eindruck; sie ist und bleibt der nächste Verwandte des nemoralis Ill.

Dagegen bin ich mehr damit einverstanden, auf Carabus auratus eine Gattung (Untergattung Autocarabus Seidlitz) zu gründen. Wir kommen nur dann zu wirklich befriedigenden Resultaten, wenn wir ungleich mehr, als es bisher geschehen, die geographische Verbreitung an die Spitze stellen und damit Reihen von Charakteren in Verbindung bringen. Ein natürliches Band um alle Carabus schlingen wollen, ist so gut wie unmöglich.

Dr. G. Kraatz. 


\section{$2 \mathrm{BHL}$ Biodiversity Heritage Library}

Kraatz, G. 1887. "Besprechung von Seidlitz' Fauna baltica ed. II." Deutsche entomologische Zeitschrift 1887(2), 361-362.

https://doi.org/10.1002/mmnd.48018870203.

View This Item Online: https://www.biodiversitylibrary.org/item/103946

DOI: https://doi.org/10.1002/mmnd.48018870203

Permalink: https://www.biodiversitylibrary.org/partpdf/235412

\section{Holding Institution}

Harvard University, Museum of Comparative Zoology, Ernst Mayr Library

\section{Sponsored by}

Harvard University, Museum of Comparative Zoology, Ernst Mayr Library

\section{Copyright \& Reuse}

Copyright Status: Public domain. The BHL considers that this work is no longer under copyright protection.

This document was created from content at the Biodiversity Heritage Library, the world's largest open access digital library for biodiversity literature and archives. Visit BHL at https://www.biodiversitylibrary.org. 